Publication du Centre de recherches sur la didacticité des discours ordinaires

16 | 2021

Analyse contrastive du discours grammatical

\title{
La compétence interprétative du locuteur dans le discours grammatical contextualisé : l'étude du genre des noms en français dans les grammaires du français conçues par les auteurs slovaques
}

Speaker's interpretative competence in contextualized grammatical discourse: a study of the gender of French nouns in French grammar books written by Slovak authors

Zuzana Puchovská

\section{(2) OpenEdition}

\section{Édition électronique}

URL : http://journals.openedition.org/cediscor/4202

DOI : $10.4000 /$ cediscor.4202

ISSN : 2108-6605

Éditeur

Presses Sorbonne Nouvelle

Édition imprimée

Pagination : 100-117

ISBN : 978-2-37906-049-6

ISSN : $1242-8345$

\section{Référence électronique}

Zuzana Puchovská, «La compétence interprétative du locuteur dans le discours grammatical contextualisé : l'étude du genre des noms en français dans les grammaires du français conçues par les auteurs slovaques », Les Carnets du Cediscor [En ligne], 16 | 2021, mis en ligne le 01 janvier 2021 , consulté le 01 février 2021. URL : http://journals.openedition.org/cediscor/4202 ; DOl : https://doi.org/ 10.4000/cediscor.4202 


\title{
La compétence interprétative du locuteur dans le discours grammatical contextualisé : l'étude du genre des noms en français dans les grammaires du français conçues par les auteurs slovaques
}

\author{
Speaker's interpretative competence in contextualized \\ grammatical discourse: a study of the gender of French nouns \\ in French grammar books written by Slovak authors
}

par Zuzana Puchovská

\section{Résumé/Abstract}

L'objectif de l'article porte sur le processus interprétatif (le processus de perception et de compréhension) des locuteurs slovaques face aux phénomènes grammaticaux du français. L'auteure pose que le discours grammatical contextualisé peut être en partie influencé par la compétence interprétative des locuteurs slovaques. Pour vérifier cette hypothèse, la première partie de l'article expose brièvement la définition et l'explication du concept linguistique de compétence interprétative que l'auteure tente de mettre en relation avec le processus d'apprentissage d'une langue étrangère ainsi qu'avec le discours grammatical contextualisé. La deuxième partie de l'article se consacre à l'analyse de la description grammaticale autour du genre des noms en français, ce qui lui permet de vérifier, dans un discours grammatical concret, la réflexion théorique préalable. Dans un premier temps, elle analyse le discours grammatical dans deux grammaires du français publiées en Slovaquie (Francúzska gramatika, Taraba 2001 et Gramatika súcasnej francúzštiny, Lingea 2011). Dans un second temps, l'auteure propose une description personnelle du genre des noms en français mettant en évidence le principe de la transparence morphosémantique qui régit le fonctionnement interne de la catégorie grammaticale du genre des noms en slovaque. Dans les deux situations, les formes de contextualisation observées sont décrites.

This article deals with the interpretative process (the process of perception and understanding) of Slovak speakers as they face French grammatical phenomena. The author asserts that contextualized grammatical discourse can be partly affected by the interpretative competence of the Slovak speakers. To check this hypothesis, the first part of the article explores the definition and explanation of the linguistic concept of interpretative competence, which the author attempts to link to the learning process of the foreign language, as well as to contextualized grammatical discourse. The second part of the article focuses on the analysis of the grammatical description of the gender of nouns in French that helps to verify the previous theoretical reflection through a concrete grammatical discourse. First, the grammatical discourse in two French grammar books published in Slovakia (Francúzska gramatika, Taraba 2001 and Gramatika súčasnej francúzštiny, Lingea 2011) is analyzed. Second, the author proposes her own description of the gender of French nouns, highlighting the principle of morphological transparency, which governs the internal functioning of the grammatical category of noun gender in Slovak. In both situations, the observed forms of the contextualization are described. 


\section{Mots-clés/Keywords}

Grammaire, discours grammatical contextualisé, compétence interprétative, genre des noms, formes de contextualisation

Grammar, contextualized grammatical discourse, interpretative competence, gender of the nouns, forms of contextualization

L présent article s'inscrit dans le champ de la didactique de la grammaire du français langue étrangère (FLE) dont la problématique a été « largement éclipsée par les méthodologies d'enseignement communicatives puis actionnelles des langues" (Beacco 2014 : 3). L'importance de la description et de l'explication grammaticales en FLE reste pourtant incontestable, comme l'affirment Besse et Porquier : « la didactique des langues ne peut contourner le problème de l'enseignement/apprentissage des régularités en particulier morpho-syntaxiques» (Besse et Porquier 1991 : 72). Dans la même optique, Beacco (2010 : 46) constate que les activités grammaticales se maintiennent largement dans l'enseignement du français. Vigner (1984 : 189) souligne également l'importance du métadiscours qui, en établissant une certaine distance entre l'apprenant et son activité, permet à celui-ci de «structurer progressivement ses acquis ». Dans cette perspective, cet article se propose d'interroger le métadiscours grammatical tel qu'il se présente dans les grammaires du français conçues et éditées hors de France. Ainsi, dans ces grammaires, les descriptions et les explications des phénomènes linguistiques se caractérisent souvent par des approches qui modifient ou bien retravaillent entièrement le discours grammatical de référence ${ }^{1}$, en s'adaptant au contexte d'enseignement du pays. Il est important de noter que l'intérêt pour la contextualisation du discours grammatical, l'intérêt pour un discours qui s'adapte en quelque sorte à la culture métalinguistique de l'apprenant ainsi qu'à ses besoins d'apprentissage, a été mis en avant par Besse et Porquier dans Grammaires et didactique des langues en 1984. Les auteurs constatent par rapport à la grammaire du français conçue par un linguiste russophone, plus précisément par rapport à la description de l'article français, que :

L'auteur s'appuie sur le modèle métalinguistique traditionnel français, mais note qu'il lui «a fallu employer des termes qui ne sont pas consacrés par l'usage et la tradition grammaticale française " (préface, p. 3). Et ce n'est que quand il s'en écarte qu'il est, de notre point de vue, le plus stimulant. [...] On voit comme cette description est à la fois plus complexe, plus nuancée, plus précise que celle de la plupart des manuels français. (Besse et Porquier 1991 : 111-112)

En effet, le discours grammatical contextualisé est par définition un discours qui s’adapte aux contextes linguistique, éducatif et culturel d'enseignement des apprenants allophones (Beacco et al. 2014; Bruley et al. 2014; Bruley et Meszaros 2014) et s'écarte du discours grammatical de référence par le souci des auteurs de rendre la description grammaticale plus accessible aux

1. Par le terme " discours grammatical de référence ", nous entendons le discours grammatical élaboré par les grammairiens et linguistes francophones qui s’adressent, dans leurs grammaires du français, en premier au public francophone (tels que Riegel et al. 2016 [1994], Grammaire méthodique du français; Grevisse et Goosse 2011 [15 édition], Le bon usage; Chevalier et Blanche-Benveniste 2002, La grammaire Larousse du français contemporain, etc.). 
besoins spécifiques des apprenants, en vue d'un apprentissage plus efficace. À titre d'exemple, on mentionne la préface du manuel édité en Slovaquie, Francuizsky jazyk pre masmediálne štúdia [Langue française pour les études des médias], où les auteurs formulent leurs objectifs de la manière suivante :

Nous avons voulu exposer le noyau de la grammaire normative du français moderne de la manière la plus claire possible. La partie théorique, les explications des phénomènes grammaticaux et des règles ainsi que leurs explications sont formulées en slovaque pour en avoir une meilleure compréhension. Les exemples sont donnés en français et sont traduits vers le slovaque. Nous avons été guidés par un simple but d'ordre pragmatique : rendre la morphologie et la syntaxe plus intelligibles (Švarbová dir. 2012 : 14, traduction de l'auteur) ${ }^{2}$.

Les auteurs de ce manuel définissent donc leur propre discours grammatical par des qualificatifs tels que la plus claire, meilleure compréhension, plus intelligible. Ces qualificatifs formulés en tant qu'un des objectifs du manuel montrent d'une part la prise de conscience des auteurs de la difficulté que peut poser la langue française aux apprenants slovaques, et laissent entrevoir, d'autre part, le souci fondamental des concepteurs du manuel de rendre l'apprentissage du français plus efficace.

Dans le cadre des recherches et des analyses du discours grammatical contextualisé ${ }^{3}$, notre intérêt porte sur les phénomènes qui engendrent ce type de discours, sur les phénomènes qui motivent et nourrissent les écarts, les variations, les modifications des descriptions grammaticales du français conçues par des auteurs non francophones. Nous pensons que, mis à part les contextes linguistique, éducatif et culturel d'enseignement local et le souci de rendre l'apprentissage plus efficace, il est important de mettre le discours grammatical contextualisé en relation avec le processus interprétatif de l'apprenant qui influencerait lui aussi les formes de contextualisation. Plus précisément, nous nous plaçons ici dans la problématique formulée par Beacco :

On s'interrogera sur le fait de savoir à quelles conditions ces descriptions didactisées sont interprétables par les apprenants et donc potentiellement utiles à leur apprentissage, c'est-à-dire sur les conditions de création de ce que l'on pourrait nommer la « qualité » du discours grammatical. $(2010: 37$ et chapitre $8: 184-215)$

C'est, en effet, la problématique de l'interprétabilité du discours grammatical contextualisé par les apprenants slovaques que nous examinerons ici, l'interprétabilité envisagée comme " condition sine qua non" du discours grammatical (Beacco 2010 : 205). Ainsi, nous nous focaliserons sur la relation entre la compétence interprétative de l'apprenant slovaque et le discours grammatical de l'enseignant, du manuel ou bien de la grammaire utilisée par celui-ci décrivant le fonctionnement des phénomènes grammaticaux du français. Nous posons que l'interprétabilité du discours grammatical contextualisé par l'apprenant pourrait augmenter à

2. Švarbová et al. 2012 : 14 : «Snahou bolo podat čo najprehl'adnejším spôsobom jadro normatívnej gramatiky modernej spisovnej francúzštiny. Teoretická čast́ a vysvetlenia gramatických javov sú písané v slovenčine pre lepšie pochopenie javov, pravidiel a ich fungovania, príklady sú uvádzané samozrejme vo francúzštine so slovenským prekladom. Sledovali sme pritom jednoduchý pragmatický ciel: sprehl'adnit učivo z morfológie a syntaxe pre slovenského učiaceho sa ".

3. Voir Présentation, note 1, p. 9. 
partir du moment où celui-ci y reconnaît le fonctionnement interne des structures grammaticales de sa langue maternelle ${ }^{4}$.

Il s'agit ici évidemment d'une hypothèse, que nous validerons dans la première partie de manière théorique en nous focalisant sur le concept de la compétence interprétative du locuteur. Dans la seconde partie, nous étudierons le genre des noms en français et nous tenterons de montrer quelles peuvent être les relations mutuelles entre le processus interprétatif $\mathrm{du}$ locuteur slovaque, régi par le fonctionnement de sa langue maternelle (pour le genre des noms en slovaque, il s'agira du principe de la transparence morphosémantique des noms) et les formes de contextualisation dans la description grammaticale de ce phénomène, c'est-àdire des variations ou des écarts par rapport à la description de référence.

Le point de départ de notre réflexion et de notre analyse est donc la langue slovaque ainsi que les locuteurs/apprenants slovaques. La langue slovaque, avec toutes ses spécificités notamment morphologiques, nous sert de prisme à travers lequel il serait possible de retravailler la description de la langue française. Ainsi, nous nous plaçons dans la perspective du concept de nativisation ${ }^{5}$, où la langue française et ses catégories grammaticales sont appréhendées à travers les structures, les catégories grammaticales et conceptuelles du slovaque.

\section{La compétence interprétative du locuteur}

L'interprétation en tant que concept notionnel est exploitée dans de nombreuses disciplines, champs de recherches ou courants, tels que la philosophie, la philosophie du langage, l'anthropologie, la sociologie, la psychologie cognitive, la linguistique ou bien la pragmatique. Dans le cadre de cet article, nous présenterons brièvement ce concept, en nous limitant à des remarques plutôt généralisantes concernant notamment le champ de la linguistique.

\subsection{La compétence interprétative dans une perspective élargie}

Nous nous appuyons principalement sur les travaux du linguiste slovaque Dolník ${ }^{6}$ (1998, 2013a, 2013b, 2014) que nous essayons de mettre en regard avec la problématique du discours

4. Sous le terme langue maternelle, nous envisagerons la/les première(s) langue(s) ou la/les langue(s) source(s) de l'apprenant. Nous sommes consciente du fait que ce terme a tendance à être de moins en moins utilisé car il ne couvre plus exactement la nouvelle réalité linguistique des apprenants (nous pensons notamment aux phénomènes du bilinguisme et plurilinguisme) ou devient « discutable dans certaines situations sociales et sociolinguistiques » (Castellotti 2001 : 21). Pourtant, nous le garderons car il évoque plus explicitement, nous semble-t-il, la relation étroite que l'apprenant crée avec la première ou les premières langues acquises, transmises ou non par la mère. Et cette relation est fondamentale pour notre réflexion.

5. Nous abordons ici la question du processus de nativisation (Andersen 1983; Ellis 1996; Demaizière et Narcy-Combes 2005; Arthaud 2007). Le processus de nativisation s'appuie sur l'approche cognitive de la capacité de l'homme à percevoir le monde qui l'entoure. En didactique des langues étrangères, ce concept souligne que l'étudiant appréhende et saisit la langue étrangère à travers les modalités et critères de sa langue maternelle, à travers sa structure et ses catégories formelles ainsi que conceptuelles. Ce processus pourrait donc plus ou moins considérablement modifier la compréhension du fonctionnement des phénomènes grammaticaux de la langue étrangère par l'apprenant.

6. Dolník, considéré comme l’un des linguistes slovaques les plus importants, se focalise dans sa recherche scientifique sur les différents aspects du langage et de la communication linguistique : les aspects culturels, politiques, sociologiques, pragmatiques et axiologiques. Il s'intéresse également à la linguistique appliquée et l'exploite dans la théorie de la traduction/interprétation ainsi que dans la transmission du slovaque en tant que langue étrangère. Dolník travaille actuellement sur la conception de la nouvelle morphologie de la langue slovaque contemporaine, sur la théorisation des principes de l'évolution et du fonctionnement de la 
grammatical contextualisé et ses formes. Nous interrogeons donc le lien possible entre la manière dont l'auteur envisage le concept linguistique de compétence interprétative du locuteur, les principes internes du fonctionnement de la langue maternelle du locuteur et les formes de contextualisation ${ }^{7}$.

Selon Dolník, la compétence interprétative ou bien le besoin d'interprétation est une qualité immanente de l'homme. Du point de vue anthropologique et philosophique, on peut dire que l'homme est un être herméneutique qui, au cours de son évolution, a toujours voulu comprendre le monde et lui attribuer un sens précisément en activant sa compétence interprétative. Autrement dit, grâce à ce processus interprétatif, l'homme est naturellement prédisposé à comprendre et à expliquer la réalité extralinguistique qui l'entoure de même que, dans une situation de communication, il est prédisposé à la compréhension de ses interlocuteurs ${ }^{8}$. Concernant le processus de lecture par exemple, Vandendorpe formule, lui aussi, cette idée : "[...] les opérations désignées par les verbes comprendre et interpréter relèvent d'une même volonté de saisir le sens " (Vandendorpe 1992 : 2). Or, il ajoute que ces deux opérations n'ont pas le même statut (ibid.) face à un texte (littéraire pour sa réflexion); il faudrait donc distinguer d'une part la compréhension du texte et d'autre part son interprétation. La vision de Dolník est plus large : il envisage la compétence interprétative du locuteur dans tous les rapports que le locuteur est susceptible d'avoir avec le monde. Dans sa réflexion, l'interprétation englobe la compréhension (même s'il distingue deux situations interprétatives que l'on peut rapprocher de la distinction compréhension/interprétation, nous le verrons plus loin) et rejoint ainsi la réflexion de Gadamer où les deux opérations sont étroitement liées : "comprendre, c'est toujours interpréter ; l'interprétation est la forme explicite de la compréhension " (Gadamer 1976 : 148, cité par Vandendorpe 1992 : 3).

D’ailleurs, le sémantisme du mot interprétation confirme la complexité du concept en question et son importance pour les locuteurs. L'étymon latin interpretari signifie traduire, expliquer, rendre compréhensible ce qui est compliqué et ambigu, saisir, comprendre, déchiffrer, représenter, etc. Il est important de noter au passage que toutes ces significations du mot interprétation se voient effectivement exploiter aussi dans le processus de l'enseignement/ apprentissage, dans le travail cognitif de l'apprenant face à la langue étrangère. Les activités mentales telles que l'explication, le déchiffrage ou la représentation par rapport à des phénomènes linguistiques de la langue étrangère font partie intégrante du processus d'apprentissage.

langue ainsi que sur la politique linguistique dans le contexte slovaque. Sa réflexion ne porte pas en soi sur la question de l'acquisition des langues étrangères (sa réflexion couvre le fonctionnement du langage humain dans sa globalité). Il nous semble pourtant intéressant d'examiner le concept de compétence interprétative du locuteur comme un des facteurs qui déterminent le " processus d'appropriation " (Véronique 2009) d'une langue étrangère en tant que capacité de l'apprenant de comprendre (interpréter) le discours grammatical de l'enseignant ou de l'auteur d'une grammaire.

7. Il est pourtant important de noter que la problématique de l'interprétation et de la compréhension est travaillée par de nombreux linguistes ainsi que philosophes. Nous voudrions mentionner par exemple la sémantique interprétative de Rastier (1987, 1996, 1998), les travaux sur la compréhension et l'interprétation dans l'activité de lecture scolaire, notamment de textes littéraires, de Grossman (1999) et Tauveron (1999) ainsi que de Vandendorpe (1992), les réflexions sur l'herméneutique de Gadamer (2011) ou de Ricœur (1997) ou encore la communication littéraire de Riffaterre (1979).

8. Gadamer insiste sur le fait que l'herméneutique est bien plus qu'une simple méthode scientifique, l'herméneutique signifie avant tout "capacité naturelle de l'homme " (Gadamer 2011 : 252). Le philosophe ajoute que cette capacité naturelle, capacité de comprendre et d'interpréter, s'active non seulement face aux textes écrits, mais aussi dans les relations que l'homme se crée avec les autres et le monde (ibid. : 275). 
Nous constatons en effet que la compétence interprétative en tant que qualité immanente de l'homme, son besoin de comprendre, sa volonté de saisir le sens se justifient également dans la façon dont les locuteurs apprennent et appréhendent par exemple les langues étrangères. Or, nous voudrions préciser que notre propos concernera uniquement la relation interprétative entre l'apprenant et le discours grammatical contextualisé.

\subsection{La compétence interprétative dans une perspective linguistique}

Pour pouvoir parler de la compétence interprétative dans la perspective linguistique, nous devons la mettre en relation avec les situations de communication où le locuteur, pour comprendre le message écrit ou oral, mobilise naturellement entre autres cette compétence. Ainsi, selon Dolník (1998 : 99-109), deux situations interprétatives s’imposent.

Premièrement, l'interprétation du message écrit ou oral se fait d'une manière naturelle; Dolník nomme alors ce processus mental interprétation naturelle (ibid. : 100-101, 108). Cela veut dire que l'identification et la compréhension du message s'effectuent spontanément et inconsciemment; on pourrait même dire automatiquement. Si l'on envisage l'acte de lecture, le concept d'interprétation naturelle rejoint le concept de compréhension fonctionnelle ou minimale (Gervais 1993) « où s'opèrent des inférences simples, souvent inconscientes et en nombre restreint pour ne pas ralentir l'avancée dans le texte » (Tauveron 1999 : 15). En d'autres termes, dans une telle situation communicative, on ne ressent pas de difficultés majeures, d'obstacles, rien ne brouille notre compréhension. Dolník (1998 : 101) constate que si le locuteur se trouve face à un message (écrit ou oral) dans sa langue maternelle, il ne peut que l'identifier et en même temps le comprendre; finalement, le locuteur reçoit le message en comprenant spontanément ce qui est dit ou écrit ${ }^{9}$. Il est nécessaire d'ajouter que le fait de comprendre un message ne signifie pas que notre compréhension de celui-ci est systématiquement correcte ou pertinente. Dans le processus d'interprétation naturelle, il s'agit de reconnaître tout simplement le contenu et le sens du message sans fournir un travail cognitif majeur. Vandendorpe (1992:2) nomme ce processus tout simplement compréhension et explique qu'il s'agit d'une part d'une opération qui «implique la saisie du sens déterminé et commun aux divers interlocuteurs » et d'autre part d'un " processus automatique " où " les relations de sens s'établissent facilement " (ibid. : 3). Le processus interprétatif naturel se déclenche, comme le constate Dolník, par le fait que le locuteur appréhende les messages avec certaines attentes ou représentations qui sont liées à ses connaissances encyclopédiques, pragmatiques, linguistiques ou métalinguistiques (1998 : 108); dans les termes de Gadamer, le locuteur appréhende le message avec une certaine "précompréhension» (2011:284). Si le message (écrit ou oral) n'est pas en contradiction avec les attentes standardisées du locuteur, on peut parler d'une situation interprétative naturelle.

La deuxième situation apparaît dès que nous sommes confrontés à des messages qui perturbent le processus interprétatif spontané et automatique, quand nous constatons face aux messages de l'opacité, une ambiguïté, une difficulté dans le sens où le message ne correspond pas à nos attentes, à nos représentations ou à notre précompréhension. Dans ce cas-là, le pro-

9. Il ne faut pourtant pas prendre l'exemple de la langue maternelle comme représentatif. Il est évident que dans la communication écrite ou orale même en langue maternelle, on rencontre des situations où la compréhension du message n'est pas assurée automatiquement et elle nécessite un travail conscient de réflexion quant au décodage du sens du message. 
cessus interprétatif devient conscient et intentionnel car nous essayons de rendre plus clair ce qui nous semble obscur pour une meilleure compréhension du message. Dolník parle d'interprétation réflexive (1998: 101) qui est une activité linguistique consciente. Vandendorpe, qui utilise dans ce cas le terme interprétation, décrit ce processus mental de la manière suivante : "L'interprétation entre en jeu lorsque la compréhension se trouve en défaut, que l'esprit est dans une impasse, qu'il bute sur un matériau irréductiblement énigmatique » (1992 : 2). Pour l'exemplifier, prenons le processus de la traduction où l'interprétation réflexive est souvent mobilisée. La traduction est une activité mentale par définition consciente et intentionnelle ; le traducteur peut être confronté à des passages du texte original dont le sens n'est pas facile à saisir, et donc son travail consiste entre autres à le déchiffrer et à le comprendre. L'activité cognitive du traducteur est liée notamment au fait qu'il est confronté à un message en langue étrangère dont le fonctionnement s'éloigne, d'une manière plus ou moins considérable, de celui de sa langue maternelle.

Pour résumer ce que l'on vient schématiquement d'exposer, on s'appuiera sur l'idée de Vandendorpe qui remarque justement que les deux opérations (interprétation naturelle ou compréhension et interprétation réflexive ou interprétation) n'ont pas le même statut, même si les deux permettent au locuteur de saisir le sens du message écrit ou oral. La différence entre ces deux opérations est liée au degré de conscience du locuteur. Ainsi, le processus interprétatif du locuteur est déterminé, précisément, par son besoin de comprendre, c'est-à-dire par sa nature d'être herméneutique. Il est en effet prédisposé à comprendre le message, sauf que cette compréhension viendrait soit spontanément, soit après un effort intellectuel et cognitif plus ou moins important.

\subsection{La compétence interprétative et l'apprentissage d'une langue étrangère}

Si nous considérons l'enseignement/apprentissage d'une langue étrangère comme une situation de communication particulière entre l'enseignant et l'apprenant ou entre l'auteur d'un discours grammatical et son récepteur, il est de rigueur de s'intéresser au rôle que peut y jouer la compétence interprétative de l'apprenant. Face à des explications ou descriptions grammaticales d'un phénomène linguistique, l'apprenant se trouve constamment dans un processus interprétatif. Or, nous pensons (en nous appuyant sur le concept de compétence interprétative de Dolník) que le locuteur, dans ce processus interprétatif, pencherait soit vers une interprétation naturelle du discours grammatical, soit vers un processus interprétatif réflexif. Autrement dit, il se peut que certaines descriptions grammaticales puissent être ressenties par l'apprenant comme opaques et difficiles à saisir ou « réticentes ${ }^{10}$ », et que d'autres puissent

10. Dans une autre perspective, celle de la lecture des textes littéraires, Tauveron utilise le concept de réticence du texte pour désigner les situations où le texte ne permet pas une compréhension immédiate et automatique du sens. La réticence, explique l'auteure, «trouve sa source dans l'ensemble des moyens qui sont utilisés, en rupture délibérée avec les lois élémentaires de la communication naturelle, pour ne pas rendre la saisie du message immédiate et laisser ainsi une part de travail au lecteur " (Tauveron 1999 : 18). Dans cette perspective, la réticence du texte (un texte qui empêche la compréhension immédiate) est envisagée comme un apport, comme une condition nécessaire pour l'apprentissage de la lecture car elle conduit les élèves à un travail plus profond de lecture. Or, dans notre perspective, celle de la conception du discours grammatical en FLE, c'est plutôt le contraire. Les descriptions ou explications grammaticales « réticentes " qui empêcheraient la compréhension immédiate de l'apprenant pourraient devenir plutôt décourageantes et ne favoriseraient pas l'appropriation de la langue étrangère par celui-ci. 
en revanche apparaître comme transparentes et faciles à comprendre. Et c'est, en effet, la conception du discours grammatical, l'explication fournie par le grammairien ou l'enseignant qui détermineraient soit l'une, soit l'autre situation interprétative. Nous constatons donc que la conception du discours grammatical entrerait en lien étroit avec le processus interprétatif de l'apprenant dans la mesure où il serait apte à le rendre davantage naturel ou au contraire davantage réflexif. Nous entrons ici dans la problématique de l'interprétabilité du discours grammatical en tant qu'un des deux critères de sa qualité, l'opérationnalité étant le deuxième critère (Beacco 2010 : 205). Nous pensons que le discours grammatical contextualisé du français deviendrait plus facilement interprétable par l'apprenant dans la mesure où il insérerait dans ses explications ou descriptions les éléments du fonctionnement interne de sa langue maternelle. En effet, la langue maternelle (la ou les langues premières) ou bien la " grammaire intériorisée $^{11}$ " de l'apprenant jouerait un rôle très important voire fondamental dans le processus d'appropriation d'une langue étrangère. Ainsi, Castellotti constate à juste titre :

On peut, dans un premier temps, décider d'ignorer ce « déjà-là » ou d'en tenir compte; on peut ensuite, si l'on accepte de reconnaître la réalité de sa présence, tenter de l'évacuer de la classe de langue étrangère, considérant qu'il fait obstacle ou, pour le moins, écran à l'appropriation d'une autre langue; on peut à l'inverse s'appuyer sur les acquis des apprentissages premiers pour les investir dans l'accès à une langue étrangère. (Castellotti $2001: 7$ )

Nous rejoignons la position de Castellotti quant à la langue maternelle de l'apprenant. Investir ce « déjà-là » dans l'accès à une langue étrangère signifierait pour nous, entre autres, l'intégrer consciemment dans la conception du discours grammatical et le rendre ainsi davantage accessible à l'apprenant. La description du fonctionnement d'une catégorie grammaticale de la langue française (par exemple, les articles, le subjonctif, le genre des noms ou bien le gérondif ou encore le futur simple) entre, en effet, dans le processus interprétatif de l'apprenant où le point de référence est représenté, naturellement, par les principes internes du fonctionnement de sa langue maternelle, c'est-à-dire du slovaque.

Ainsi, nous postulons que si le discours grammatical s'appuie sur les principes internes $\mathrm{du}$ fonctionnement du slovaque, il devient contextualisé et, par conséquent, le processus interprétatif des apprenants deviendrait plus naturel. Lappropriation ainsi que la maîtrise du phénomène grammatical viendraient donc plus spontanément. En revanche, si le discours grammatical ne prend pas ou très peu en considération ces principes, s'il n'est pas ou très peu contextualisé, l'apprenant mettrait ainsi en activité l'interprétation réflexive et l'appropriation viendrait après un effort intellectuel plus important ${ }^{12}$. Notre hypothèse voudrait donc mettre en relief le rapport qui existe entre les principes internes du fonctionnement de la langue

11. La notion de grammaire intériorisée est problématisée et analysée notamment par Besse et Porquier dans l'ouvrage Grammaires et didactique des langues (1993 : 13-16). Il s'agirait d'un phénomène proprement humain, d'ordre bio-génétique et psycho-social, relevant donc à la fois de l'inné et de l'acquis. Il s'agirait d'une connaissance implicite du système de la langue, celle-ci acquise par un processus inconscient de formation, de vérification et de traitement des données de la langue en question. Enfin, elle serait à la fois individuelle (la base des idiolectes propres à un locuteur) et collective (présentant des caractéristiques communes à l'ensemble des locuteurs).

12. Dans cette perspective, on pense immédiatement à la notion de transfert positif (les similitudes) entre les langues qui favoriserait l'apprentissage et à la notion d'interférence (les différences) entre les langues qui est souvent la cause des erreurs chez l'apprenant. Il ne s'agit pas de chercher uniquement des similitudes et des différences entre le français et le slovaque; il s'agit d'interroger les principes internes du fonctionnement de la 
maternelle de l'apprenant, le discours grammatical qui s'en inspire et le processus interprétatif de l'apprenant.

\section{2. Étude du genre des noms en français}

Dans cette seconde partie, nous expliciterons nos réflexions théoriques par un exemple précis, celui du genre des noms en français. Tout d'abord, nous nous intéresserons à la description de la catégorie du genre des noms telle qu'elle se présente dans les discours grammaticaux des auteurs slovaques. Nous analyserons deux grammaires, celle de Taraba Francúzska gramatika [Grammaire française] (2001, 1995 pour la $1^{\text {re }}$ édition) et celle du collectif d'auteurs de la maison d'édition Lingea qui est une traduction adaptée de la version tchèque de cette grammaire, Gramatika súčasnej francúzśtiny [Grammaire du français contemporain] (2011) ${ }^{13}$. Ce qui nous intéressera, c'est comment la description du phénomène en question y est menée et si les principes internes du fonctionnement de la catégorie grammaticale du genre des noms en slovaque se reflètent dans le discours grammatical des auteurs, autrement que par le processus d'analyse contrastive du phénomène. Ensuite, nous terminerons notre propos par la proposition d'une description grammaticale personnelle du genre des noms en français, qui s'appuiera consciemment sur le principe de la transparence morphosémantique des noms en slovaque et mettra ainsi en valeur la situation interprétative naturelle des apprenants slovaques.

\subsection{Analyse du discours grammatical dans les grammaires du français éditées en Slovaquie}

L'enseignement et l'apprentissage de la catégorie grammaticale du genre des noms en français montrent en principe deux difficultés majeures pour les apprenants du FLE. Il s'agit d'abord pour l'apprenant de savoir quel est le genre du nom en question (masculin ou féminin) comme le constate De Salins (1996 : 46) en ajoutant qu'« il n'existe malheureusement pas de règle infaillible pour connaître le genre des noms en français. [...] On conseille habituellement aux apprenants de mémoriser le nom et son article. »On verra que le discours grammatical contextualisé pourrait apporter certaines solutions à cette difficulté. Ensuite, l'apprenant est amené à comprendre le principe de la formation du genre féminin à partir du masculin. La catégorie du genre des noms concerne donc notamment le fonctionnement morphologique de la langue, ce qui est largement retenu dans le discours grammatical observé dans les deux grammaires du français étudiées ${ }^{14}$.

langue maternelle de l'apprenant et comment ceux-ci se reflètent dans le discours grammatical contextualisé du français.

13. La maison d'édition Lingea ne précise pas les auteurs de la version slovaque de cette grammaire. Elle mentionne uniquement que la "grammaire a été élaborée par le collectif d'auteurs des employés de Lingea ».

14. Il faudrait pourtant insister sur le fait que le genre des noms en français représente un phénomène complexe et son fonctionnement dépend de la nature du nom en question. On envisage la problématique du genre différemment pour les noms propres et les noms communs, pour les noms animés et les noms non animés ainsi que pour les noms simples et les noms composés. Même si nous pouvons être d'accord avec l'idée qu' " on doit prendre le genre d'un nom comme une donnée lexicale et l'apprendre sans y réfléchir outre mesure » (Weinrich 1989 : 32) ou qu'il "suffira donc d'indiquer les grandes régularités morphologiques [...] que la classe nominale manifeste à travers les catégories du genre et du nombre " (Riegel et al. 2002 : 172), nous pensons que le discours grammatical contextualisé est susceptible de systématiser le fonctionnement de cette catégorie. 


\subsubsection{Francúzska gramatika (Taraba 2001 : 18-25) ${ }^{15}$}

Concernant le traitement du genre des noms en français, Taraba présente le phénomène grammatical en quatre temps, en quatre chapitres courts : 1) le genre des noms, 2) les noms à double genre, 3) la formation du féminin et 4) les particularités de la formation du féminin. Le discours grammatical dans ces quatre chapitres se base notamment sur le critère formel; l'auteur appuie son explication sur l'aspect morphologique du genre des noms :

[1] Comme le genre est dans ce cas motivé par l'origine étymologique des mots, on peut le distinguer plus facilement à l'aide des signes formels typiques. (Taraba 2001 : 18, notre traduction) ${ }^{16}$

[2] Les mots d'origine latine et grecque qui s'utilisent en slovaque ainsi qu'en français ont le même genre ou un genre différent. L'interférence peut être causée par le fait qu'en français ces mots se terminent souvent par -e (signe du genre féminin). (Taraba $2001: 19$, notre traduction) ${ }^{17}$

[3] En français, il existe trois façons de former le pendant féminin du genre masculin. (Taraba 2001 : 22 , notre traduction) ${ }^{18}$

Les trois exemples cités montrent clairement la tendance de Taraba d'expliquer le fonctionnement du genre des noms en français par son aspect morphologique; notons les expressions signes formels typiques en [1], signe du genre féminin en [2] ou trois façons de former en [3] qui relèvent du métalangage morphologique traditionnel tel que d'autres termes (terminaison, suffixe, racine, voyelle radicale) observés dans le discours du linguiste. Il nous semble que dans ce sens l'approche de Taraba ne s'éloigne pas particulièrement des grammaires de référence, puisque dans ces dernières l'aspect formel du genre des noms est aussi privilégié.

Ce qui change en revanche, et on y voit apparaître effectivement des formes de contextualisation, ce sont des descriptions plus détaillées, plus systématisées de certains phénomènes liés au genre des noms en français. Il s'agit notamment des phénomènes que le slovaque ne connaît pas ou très peu, à savoir le changement sémantique du nom par rapport à son genre. Ainsi dans le chapitre "Les noms à double genre", on constate un type de contextualisation déjà observé dans la grammaire de Taraba par Bruley et Meszaros (2014 : 13), à savoir la modification de la description ou de la catégorie grammaticale ${ }^{19}$. Pour saisir plus systématiquement le phénomène quasi inexistant en slovaque, Taraba modifie la description grammaticale sans introduire pour autant une nouvelle appellation ${ }^{20}$; le linguiste crée trois catégories de

15. Taraba a conçu sa grammaire pour un public plus restreint et plutôt académique, ce qui lui a permis de travailler les phénomènes grammaticaux en profondeur en évoquant notamment les connexions d'ordre étymologique et historique avec le fonctionnement actuel du français.

16. «Kedže rod je v tomto prípade výsledkom etymologického pôvodu slov, najlahšie sa rozlišuje podl'a typických formálnych znakov. » (Taraba $2001: 18$ )

17. "Slová latinského a gréckeho pôvodu, ktoré sa používajú v slovenčine aj vo francúzštine, majú rovnaký alebo odlišný rod. Zdrojom interferencie môže byt práve fakt, že vo francúzštine sú väčšinou zakončené na -e (znak ženského rodu). " (Taraba $2001: 19)$

18. "Vo francúzštine existujú tri spôsoby, ako možno utvorit k mužskému rodu jeho ženský náprotivok. " (Taraba $2001: 22$ )

19. Les auteurs ont recensé plusieurs types de contextualisation dans la grammaire de Taraba, tels que la comparaison négative ou positive, le regroupement inhabituel de deux faits linguistiques et les traductionsléquivalences (Bruley et Meszaros 2014). Certains types de contextualisation se vérifient aussi dans la description grammaticale du genre des noms.

20. Pour la contextualisation de type modification de la description ou de la catégorie grammaticale, on observe différents degrés : «l'appellation de la catégorie est modifiée mais le contenu qu'elle désigne reste identique; l'appellation et le contenu d'une catégorie sont transformés et dans ce cas on découpe autrement la langue. Enfin, une nouvelle catégorie est inventée [...]»(Bruley et al. 2014 : 16). 
noms à double genre et apporte ainsi une modification de la description de référence qui permet une meilleure orientation par rapport au phénomène grammatical : 1) les noms où le changement du genre n'influence pas le changement sémantique, 2) les noms où le changement du genre influence partiellement le changement sémantique et 3) la fonction distinctive du genre grammatical (Taraba, 2001 : 19-21). Notons encore que la problématique des noms à double genre est dans la grammaire de Taraba exposée en trois pages avec de nombreux exemples illustratifs, tandis que dans la grammaire de Riegel et al., par exemple, ce phénomène est couvert par une seule phrase ${ }^{21}$. La modification de la description grammaticale par rapport aux noms à double genre ainsi que l'explication grammaticale plus étendue de ce phénomène signalent la volonté du grammairien d'exposer le phénomène grammatical quasi inexistant en slovaque en vue de sa compréhension par les apprenants. À partir du moment où Taraba décide de développer la problématique des noms à double genre et surtout d'en proposer une catégorisation personnelle, on constate un travail de contextualisation conscient qui prend en considération la langue maternelle des apprenants et les difficultés (notamment au niveau de la compréhension) qu'ils peuvent ressentir face aux noms à double genre en français. Or, cette contextualisation est motivée, semble-t-il, uniquement par l'analyse contrastive et ne s'appuie pas véritablement sur le fonctionnement interne de la langue slovaque.

Il est également intéressant de remarquer que Taraba se focalise uniquement sur le genre des noms communs. Le genre des noms propres, surtout des noms géographiques n'est ni expliqué, ni mentionné et on rencontre la même situation pour la question du genre des noms composés. Pourtant, il s'agit de deux aspects très importants pour la bonne maîtrise du français. Ainsi, dans son discours grammatical, Taraba opère des choix personnels; son discours se caractérise par des réductions ou bien des omissions des phénomènes pourtant présents dans le discours grammatical de référence.

Globalement, dans son discours grammatical, Taraba privilégie le fonctionnement morphologique du genre des noms et développe considérablement ce phénomène langagier inexistant en slovaque. Enfin, nous avons pu observer les formes de contextualisation suivantes : 1) les surdescriptions (notamment au niveau des phénomènes grammaticaux inexistants en langue maternelle) 2) la modification de la description grammaticale 3) l'adaptation au contexte linguistique et métalinguistique de l'apprenant et 4) les traductions et les équivalences.

\subsubsection{Gramatika súčasnej francúzštiny (Lingea 2011 : 17-20)}

Gramatika súčasnej francúzštiny [Grammaire du français contemporain] est une grammaire qui est destinée plutôt au grand public et à des non-spécialistes, ce qui se reflète immédiatement dans la conception de l'explication du genre des noms en français : l'exposé du phénomène se fait en trois pages de petit format (A6), les explications sont réduites au profit de nombreux exemples et le discours grammatical des auteurs se limite à la transmission des règles nécessaires pour maîtriser ce phénomène :

21. Bon nombre d'homonymes, par ailleurs, ne se distinguent que par le genre : le livre/la livre; le moule/la moule; le vasella vase; le mort/la mort, etc. (Riegel et al. 2002 : 172) 
[4] Dans certains cas, le genre des noms est indiqué par sa terminaison ou son suffixe. (Lingea $2011: 17$, notre traduction) ${ }^{22}$

[5] Pour certains noms de genre féminin, on observe la double consonne ou le changement de la consonne finale. (Lingea $2011: 19$, notre traduction) ${ }^{23}$

[6] Certains noms ont parfois la même forme pour le masculin et pour le féminin. (Lingea 2011 :

19 , notre traduction) $)^{24}$

On remarque donc une explication très brève ciblée sur l'information essentielle, et qui porte exclusivement sur le fonctionnement formel, morphologique du genre des noms en français. De même que pour Taraba, le métalangage traditionnel est fortement présent dans cette grammaire (terminaison, suffixe, double consonne, consonne finale, forme, radical, etc.). L'aspect formel du discours grammatical est visible notamment dans la conception de la description du genre des noms qui se fait en deux chapitres : 1 ) « le genre des noms » (les auteurs expliquent le fonctionnement des noms non animés et des suffixes qui le déterminent) et 2) «le féminin » (le chapitre est consacré à la formation du féminin). Enfin, les auteurs complètent la problématique par les noms à double genre en faisant une liste de noms avec leurs traductions en slovaque sans aucune remarque théorique. Ainsi, le discours grammatical des auteurs de Gramatika súčasnej francúzštiny simplifie au maximum la description de référence dans le but de ne pas complexifier le phénomène linguistique. On devrait d'ailleurs se demander ici, et surtout vérifier sur le terrain, si le discours grammatical qui se caractérise par la simplification et la réduction de l'explication grammaticale au strict minimum ne favoriserait pas davantage le processus interprétatif de l'apprenant et s'il ne garantirait pas en quelque sorte une meilleure maîtrise par l'apprenant des phénomènes grammaticaux, comme le préconisent les auteurs (Lingea $2011: 7$ ).

Enfin, il est intéressant de noter que, dans la grammaire de Lingea, l'explication ne concerne que les noms communs comme dans le cas de la grammaire de Taraba. Les noms propres (les noms de pays) sont mentionnés seulement dans la partie consacrée à la préposition " en " sans évoquer le fonctionnement du genre pour ce type de noms. Dans ce cas, on peut se demander si parler ici d'une forme de contextualisation, à savoir le regroupement inhabituel de deux faits linguistiques, est pertinent puisque dans les manuels de FLE de référence, on peut trouver le même type de regroupement :

[7] en : les noms de pays et de régions au féminin et les noms de pays au masculin qui commencent par une voyelle $\rightarrow$ en Belgique, en Iran, en Provence, en Anjou. (Lingea 2011 : 161, notre traduction $)^{25}$

Dans l'ensemble, le discours grammatical de Lingea privilégie le fonctionnement morphologique du genre des noms en français et propose des explications grammaticales limitées à des informations nécessaires; on observe également des absences d'explication grammaticale au profit des seuls exemples. On constate que le discours grammatical ne s'appuie pas sur

22. «V niektorých prípadoch je rod podstatného mena naznačený jeho zakončením alebo príponou. » (Lingea $2011: 17)$

23. " Pri niektorých podst. menách ženského rodu nastáva zdvojenie alebo zmena koncovej spoluhlásky. " (Lingea $2011: 19$ )

24. « Niektoré podstatné mená majú rovnaký tvar v mužskom aj ženskom rode. » (Lingea 2011 : 19)

25. « en: názvy krajín a regiónov v ženskom rode a názvy krajín v mužskom rode začínajúce sa na samohlásku: en Belgique, en Iran, en Provence, en Anjou. » (Lingea 2011 : 161) 
le fonctionnement interne de la langue slovaque; au final, on le considère comme très peu contextualisé. Enfin, nous pouvons mentionner surtout les contextualisations de type :
1) descriptions grammaticales réduites
2) traductions et équivalences dans la représentation en miroir
3) regroupement inhabituel de deux faits linguistiques.

\subsection{Le discours grammatical contextualisé et le fonctionnement interne de la catégorie du genre en slovaque}

D'après l'étude de la grammaire de Taraba et celle de Lingea destinées aux apprenants slovaques, on peut constater que le discours grammatical des auteurs s'appuie notamment sur l'aspect morphologique du genre des noms en français et sur un métalangage traditionnel connu par les apprenants slovaques. Comme la catégorie grammaticale du genre des noms est dans les deux langues une catégorie morphologique, cette observation n'est pas particulièrement surprenante.

Nous sommes d'avis pourtant que les deux discours grammaticaux analysés n'ont pas examiné véritablement le fonctionnement interne du slovaque par rapport à la catégorie du genre des noms et qu'on pourrait l'exploiter ou l'investir davantage dans la conception d'une description contextualisée plus adaptée au processus interprétatif des apprenants.

Pour cela, il faut savoir qu'en slovaque le genre des noms est régi par le principe de la transparence morphosémantique, ce qui veut dire que c'est la structure formelle du nom qui reflète d'une manière plus ou moins transparente le trait sémantique féminin ou masculin. Autrement dit, la reconnaissance du genre se fait en slovaque principalement au niveau de la forme morphologique du nom. Ainsi, les apprenants slovaques s'approprieraient plus naturellement les cas où ils identifieraient en français le même principe de fonctionnement car cela correspondrait à leurs intuitions et savoirs linguistiques.

Par conséquent, concernant le genre des noms en français, il est possible de créer une catégorisation ou encore une description grammaticale qui repose sur ce principe de fonctionnement du genre des noms en slovaque et aide ainsi les apprenants slovaques à mieux intérioriser ce phénomène. Nous proposons en effet deux cas de figure : le premier se caractérise par le fait que le genre des noms est indiqué par la seule structure morphologique du nom et le second souligne le rôle fondamental du déterminant dans le marquage du genre des noms en français ${ }^{26}$. En conséquence, notre description grammaticale du genre des noms en français exposera le phénomène en deux grandes catégories. Nous précisons qu'il ne s'agit ici que d'une réflexion, d'une esquisse de cette description grammaticale contextualisée, pour montrer les possibilités qu'ouvre le discours grammatical contextualisé au moment où nous y insérons consciemment les éléments du fonctionnement interne de la langue maternelle de l'apprenant.

\subsubsection{Indicateur du genre - structure morphologique du nom}

En slovaque, les noms appartiennent à des modèles de déclinaison du genre féminin, masculin et neutre, selon leur terminaison. La terminaison du nom ou bien sa structure morpholo-

26. Il est à noter que cette catégorisation concerne la forme écrite du français. Il est évident que, pour la forme orale, cette catégorisation ne sera pas en vigueur. 
gique est le premier indicateur du genre en slovaque. Les apprenants slovaques acquièrent au fur et à mesure de leur scolarité des connaissances théoriques et métalinguistiques de ces modèles de déclinaison sur lesquelles ils appuient leurs savoirs et représentations linguistiques. Concernant les noms en français, nous pensons qu'il existe de tels cas où l'indicateur du genre correspond à leur structure morphologique. Dans le discours grammatical que nous proposons, nous soulignerons donc ce fait et nous orienterons les apprenants vers la prise en compte de la forme morphologique du nom pour reconnaître son genre grammatical. Il nous semble qu'ainsi la description contextualisée favoriserait le processus interprétatif naturel de l'apprenant et n'empêcherait pas la compréhension immédiate du message, ce qui conduirait à une appropriation plus spontanée du phénomène grammatical. Dans cette catégorie, nous mettrons quatre groupes de noms dont la structure morphologique reflète le trait sémantique " masculin » et le trait sémantique "féminin " de même qu'en slovaque ${ }^{27}$ :

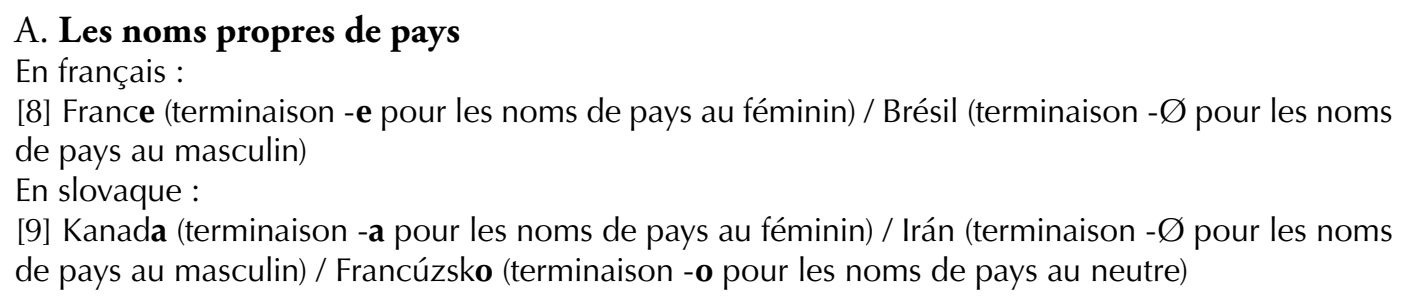

\section{B. Terminaisons marquant le féminin et le masculin des noms communs non animés} En français :

[10] voyage, avis, fantasme, document, soir (suffixes masculins) / promesse, valeur, question, histoire, boulangerie, société, culture, balade, maison, résidence (suffixes féminins) En slovaque :

[11] rýchlik, realizmus, koberec, kurín (suffixes masculins) / voda, pekáreň, možnost', živica, učebňa (suffixes féminins) / slovo, pole, pohorie, detstvo, operadlo (suffixes neutres)

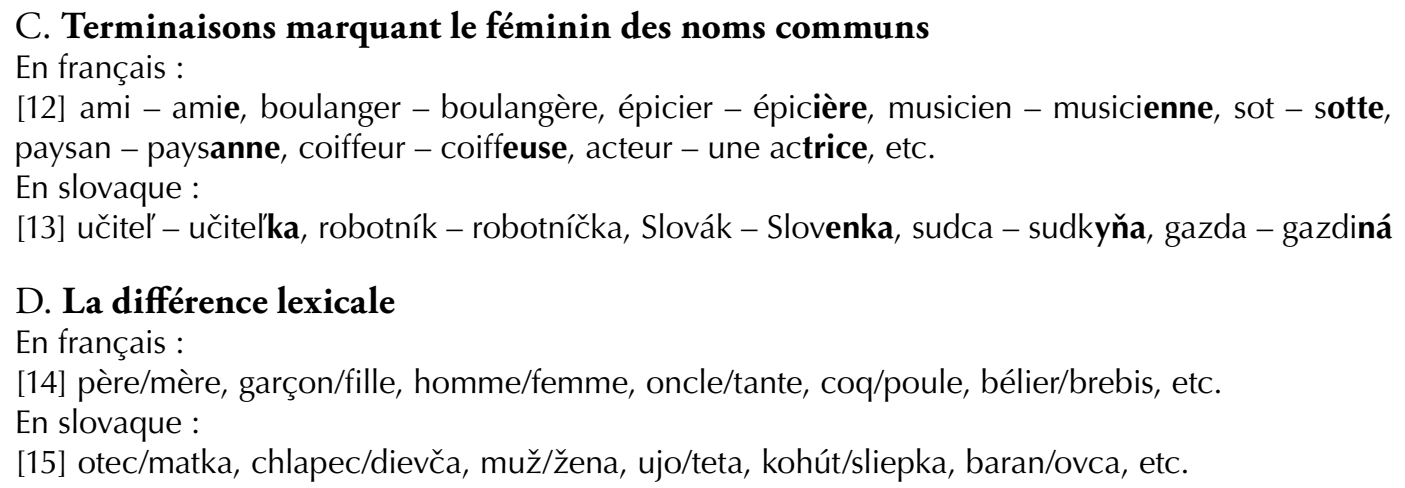

\subsubsection{Indicateur du genre uniquement par le déterminant}

En français, on observe, évidemment, des cas où la structure morphologique du nom ne reflète pas ou très peu le trait sémantique " féminin » ou " masculin ». Lapprenant slovaque ne peut ainsi pas deviner le genre du nom à partir de sa forme morphologique, comme il en serait capable avec les noms mentionnés plus haut. Par conséquent, il lui faudrait focaliser son

27. Nous sommes parfaitement consciente qu'il existe des exceptions à la règle et que notre catégorisation ne doit pas être présentée comme close. D’ailleurs, l'exception est un phénomène linguistique auquel les apprenants sont, en général, habitués et qui ne leur pose pas de grandes difficultés au niveau de la compréhension. 
attention surtout sur l'emploi du déterminant qui accompagne le nom et qui indique explicitement son genre et son nombre et apprendre pour ainsi dire le genre du nom par cœur. Ainsi, dans cette explication du fonctionnement du genre des noms en français, nous sensibiliserons l'apprenant slovaque au rôle très important que jouent l'article et les autres déterminants dans la langue française. La nécessité d'emploi de l'article ou d'un autre déterminant devant le nom se verrait ainsi justifiée et renforcerait en même temps l'acquisition d'un phénomène linguistique que la langue maternelle de l'apprenant ne connaît pas. Ajoutons que le discours grammatical contextualisé rendrait de nouveau le processus interprétatif plus naturel. En effet, nous fondons toujours ce discours grammatical sur le fonctionnement interne de la langue slovaque; or pour la présente description, nous procédons par comparaison négative. Dans cette catégorie, nous mettrons quatre groupes de noms dont la structure morphologique ne reflète pas le trait sémantique " masculin " ni le trait sémantique "féminin ".

\section{A. Les noms communs animés à un seul genre}

[16] un modèle, un témoin, un usager / une connaissance, une personne, une vedette, une victime

\section{B. Les noms épicènes}

[17] un / une camarade, un / une collègue, un / une touriste, un / une journaliste, un / une artiste, etc.

\section{Les noms à double genre}

[18] un / une mode, un / une crêpe, un / une livre, un / une physique, un / une mémoire, etc.

\section{Les noms composés}

[19] un petit-beurre, une voiture-restaurant, un chef-d'œuvre, un après-guerre, une arrière-pensée, etc.

Pour conclure, nous constatons que la relation étroite entre le fonctionnement interne de la catégorie du genre des noms en slovaque, la compétence interprétative de l'apprenant et le discours grammatical est, dans le cas du genre des noms, possible et intéressante. Nous avons ainsi pu créer une nouvelle catégorisation du phénomène grammatical qui retravaille sensiblement et le discours grammatical de référence et le discours grammatical déjà contextualisé dans les grammaires slovaques étudiées. Avec ce discours grammatical, nous établissons deux catégories de noms en français par rapport à leur structure formelle et nous leur attribuons des traits communs : l'aspect morphosémantique pour les uns, et l'importance du déterminant pour les autres. Notre discours grammatical ne se limite donc pas seulement à souligner les phénomènes inexistants en slovaque (les noms à double genre par exemple) et à évoquer les règles de formation du féminin en français; il tente de systématiser la description selon le principe de la transparence morphosémantique, principe fondamental pour la morphologie slovaque. Le discours grammatical devient ainsi contextualisé et facilite l'appropriation du phénomène grammatical dans la mesure où les apprenants se trouvent dans une situation interprétative naturelle. Plusieurs éléments sont ainsi mis en exergue :

- Le genre des noms en français peut être acquis par l'apprenant slovaque sans nécessairement passer par un processus de mémorisation obligé.

- Procéder à une description des formes conjointes par leur structure formelle peut faciliter leur apprentissage et la compréhension du fonctionnement du genre en français. 
- Le phénomène grammatical quasi inexistant en slovaque (les noms à double genre, les noms épicènes et le genre des noms composés) est intégré dans une logique d'apprentissage plus adaptée à l'apprenant.

- La compréhension de l'importance des déterminants en français est renforcée par la description contextualisée du fonctionnement du genre des noms.

Nous voudrions terminer notre propos en nous appuyant sur les travaux de HoudebineGravaud concernant le concept d'imaginaire linguistique qui permet d'étudier le « rapport du sujet à la langue, la sienne et celle de la communauté qui l'intègre comme sujet parlant-sujet social ou dans laquelle il désire être intégré, par laquelle il désire être identifié par et dans sa parole [...]» (2005: 10). Le rapport à sa langue maternelle (par ses attitudes évaluatives, fictives, prescriptives, communicationnelles et identitaires) devient, pour le locuteur, fondamental. Vaňková, linguiste tchèque, le confirme quand elle souligne que c'est dans la langue maternelle qu'on se sent chez soi et ajoute que la langue maternelle représente pour nous le point de départ, le lien avec la mère, la famille, le pays. Elle représente aussi la norme, la sécurité et elle est la base de notre relation au monde (2007 : 17). Or, il est très important de souligner que ce rapport se reflète dans le rapport que l'apprenant se construit avec la langue étrangère, et cela par exemple dans la mesure où l'apprenant projette les représentations linguistiques et métalinguistiques de sa langue maternelle sur les structures de la langue étrangère. Comme s'il cherchait, nous avons envie de dire, sa langue maternelle dans la langue étrangère, qu'il est en train d'apprendre, pour se rassurer. Dans cette perspective, la compétence interprétative du locuteur (telle que la conçoit Dolník et qu’on a tenté d'appliquer à cette étude) s'avère intéressante car elle montre justement que les situations interprétatives naturelles sont favorisées notamment quand les représentations et attentes de l'apprenant ne sont pas en contradiction avec le message qu'il reçoit, quand il se sent en quelque sorte rassuré. Ainsi, le discours grammatical contextualisé, qui s'appuie sur le fonctionnement interne de la langue maternelle de l'apprenant, est en mesure de générer ce type de situation et peut contribuer à la meilleure appropriation et maîtrise des phénomènes grammaticaux.

\section{Éléments bibliographiques}

Andersen, Roger, 1983, Pidginization and Creolization as Language Acquisition, Rowley, Newbury House.

Arthaud, Paul, 2007, Création et utilisation de ressources pédagogiques sur support numérique pour l'enseignement de l'anglais dans une école d'ingénieurs. Modalités d'intégration et étude d'impact. Éducation, https://tel.archives-ouvertes.fr/tel-00177343/document.

BEACCO, Jean-Claude, 2010, La didactique de la grammaire dans l'enseignement du français et des langues, Paris, Didier.

Beacco, Jean-Claude, Kalmbach, Jean-Michel et Suso Lopez, Javier, 2014, Les contextualisations de la description du français dans les grammaires étrangères : présentation, Langue française 181, Paris, Armand Colin : 3-17. 
Besse, Henri et Porquier, Rémy, 1991, Grammaires et didactique des langues, Paris, Didier, 1993.

Bruley, Cécile, Fouillet, Raphaëlle, Stratilaki-Klein, Sofia et Weber, Corinne, 2014, Grammaires du français et discours grammaticaux contextualisés, dans Aguilar, J., Brudermann, C., Leclere, M., dir., Complexité, diversité et spécificité : pratiques didactiques en contexte, Université Sorbonne Nouvelle - Paris 3 : 11-24.

Bruley, Cécile et Meszaros, Branislav, 2014, Grammaire du français et discours grammatical contextualisé : le cas des grammaires éditées en Slovaquie, Philologia 12/2, Bratislava, Univerzita Komenského : 7-20.

Bruley, Cécile et Starościak, Katarzyna, 2014, L'aspect accompli en français et dans les langues slaves : quels rapprochements dans les grammaires du français éditées en Pologne et en Slovaquie?, Langue française 181 : 37-57.

Castellotti, Veronique, 2001, La langue maternelle en classe de langue étrangère, Paris, Clé International.

Charaudeau, Patrick, 1992, La grammaire de sens et de l'expression, Paris, Hachette.

Chevalier, Jean-Claude et Blanche-Benveniste, Claire, 2002, La grammaire Larousse du français contemporain, Paris, Éditions Larousse.

De Salins, Geneviève-Dominique, 1996, Grammaire pour l'enseignementlapprentissage du Fle, Paris, Didier/Hatier.

Demaizière, Françoise et Narcy-Combes, Jean-Paul, 2005, Méthodologie de la recherche didactique : nativisation, tâches et TIC, Apprentissage des Langues et Systèmes d'Information et de Communication (Alsic) 8, Paris, Adalsic : 45-64.

Dolník, Juraj, 2012, Sila jazyka [Pouvoir de la langue], Bratislava, Kalligram.

DoLník, Juraj, 2013a, Interpretácia ako konštituent človeka, komunikácie a porozumenia [Interprétation comme constituant de l'homme, de la communication et de la compréhension], Jazykovedný časopis 64/2, Bratislava, SAV : 93-108.

DoLník, Juraj, 2013b, Všeobecná jazykoveda [Linguistique générale], Bratislava, VEDA.

DoLník, Juraj, 2014, Interpretácia a autonomizácia [Interprétation et autonomisation], dans Orgoňová, O., Muziková, K. et Popovičová Sedláčková, Z., dir., Jazyk a jazykoveda v interpretácii 2, Bratislava, Univerzita Komenského : 21-30.

Dolník, Juraj et BAJzíKovÁ, Eugénia, 1998, Textová lingvistika [Linguistique textuelle], Bratislava, Stimul.

Dvonč, Ladislav, dir., 1966, Morfológia slovenského jazyka [Morphologie de la langue slovaque], Bratislava, SAV.

Ellis, Rod, 1996, The Study of Second Language Acquisition, Oxford, Oxford University Press.

Gadamer, Hans-Georg, 2011, Pravda a metoda II [Vérité et Méthode II], Praha, Triada.

Gervais, Bertrand, 1993, À l'écoute de la lecture, Montréal, VLB éditeur.

Grevisse, Maurice et Goosse, André, 2011, Le bon usage, Bruxelles, Éditions De Boeck. 
Grossman, Francis, 1999, Littératie, compréhension, interprétation des textes, Repères 19 : 139-166.

Houdebine-Gravaud, Anne-Marie, 2005, L'Imaginaire linguistique, Paris, L'Harmattan.

LingeA, dir., 2011, Gramatika súčasnej francúzštiny, Bratislava, Lingea.

Rastier, François, 1987, Sémantique interprétative, Paris, PUF.

Rastier, François, 1996, Représentation ou interprétation? Une perspective herméneutique sur la médiation sémiotique, dans Rialle, V. et Fisette, D., Penser l'esprit : des sciences de la congnition à une philosophie cognitive, Grenoble, PUG : 219-239.

RAstier, François, 1998, Le problème épistémologique du contexte et le statut de l'interprétation dans les sciences du langage, Langage 129 : 97-111.

Ricceur, Paul, 1997, Teória interpretácie: Diskurz a prebytok významu [Théorie de l'interprétation : Discours et surplus de sens], Bratislava, Archa.

Riegel, Martin, Pellat, Jean-Christophe et Rioul, René, 2002, Grammaire méthodique du français, Paris, PUF.

Riffaterre, Michael, 1979, La production du texte, Paris, Seuil.

ŠvarbovÁ, Eva, dir., 2012, Francúzsky jazyk pre masmediálne štúdiá [Langue française pour les études des médias], Nitra, Univerzita Konštantína Filozofa.

TARABA, Ján, 2001, Francúzska gramatika [Grammaire française], Bratislava, SPN.

Tauveron, Catherine, 1999, Comprendre et interpréter le littéraire à l'école : du texte réticent au texte proliférant, Repères 19 : 9-39.

Vandendorpe, Christian, 1992, Comprendre et interpréter, dans Préfontaine, C. et Lebrun, M., dir., La lecture et l'écriture. Enseignement et apprentissage, Montréal, Éditions Logiques : 159-181.

VAŇKová, Irena, 2007, Nádoba plná řeči, Praha, Karolinum.

VéroniQue, Daniel, 2009, L'acquisition de la grammaire du français langue étrangère, Paris, Didier.

Vigner, Gérard, 1984, L'exercice dans la classe de français, Paris, Hachette.

WeInRICH, Harald, 1989, Grammaire textuelle du français, Paris, Didier/Hatier.

\section{L'AUTEURE}

Zuzana Puchovská est maître de conférences au Département d'études romanes à l'université Comenius de Bratislava (Slovaquie) et ses intérêts pédagogiques ainsi que scientifiques portent sur le domaine de l'enseignement de la grammaire (notamment de la morphosyntaxe) aux futurs traducteurs/interprètes et enseignants, dans une perspective contrastive françaisslovaque. Depuis 2015, elle participe aux travaux du groupe de chercheurs Grammaire et contextualisations et son travail s'oriente vers les problématiques du discours grammatical contextualisé pour le public slovaque. 